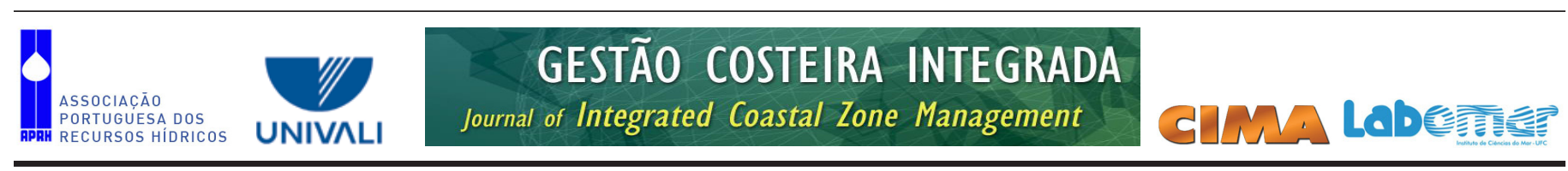

http://www.aprh.pt/rgci/pdf/rgci-377_Ribeiro.pdf | DOI:10.5894/rgci377

\title{
Evolução da vulnerabilidade à erosão costeira na Praia de Massaguaçú (SP), Brasil *
}

\section{Evolution of vulnerability to coastal erosion at Massaguaçú Beach, Brazil}

\author{
Juliana dos Santos Ribeiro ${ }^{\circledR,}$, , Paulo Henrique Gomes de Oliveira Sousa ${ }^{1}$, \\ Danilo Rodrigues Vieira ${ }^{1}$, Eduardo Siegle ${ }^{1}$
}

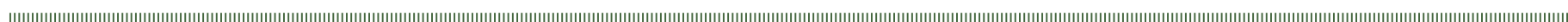

\section{RESUMO}

A erosão costeira pode causar grandes prejuízos tanto ambientais quanto sociais nas áreas atingidas e a ocupação humana mal planejada nesses locais pode agravar o processo erosivo. A praia de Massaguaçú, localizada no litoral norte do Estado de Sáo Paulo, vem sofrendo processos erosivos intensos nos últimos anos. O presente estudo teve como objetivo analisar a vulnerabilidade à erosão da praia em questão nos últimos 40 anos a partir de nove indicadores de vulnerabilidade: largura da praia, posição da linha de costa, configuraçóes ao largo, presença de rios e/ou desembocaduras, elevação do terreno, vegetação, obras de engenharia costeira, taxa de ocupação e permeabilidade do solo. Os resultados mostraram que a vulnerabilidade à erosão náo teve um aumento constante ao longo do período analisado. A parte central de Massaguaçú, hoje alvo de um processo erosivo intenso, foi a que apresentou a maior vulnerabilidade à erosão ao longo do tempo. Apesar de a ocupação humana aparentemente ter tido participaçáo nos resultados, concluiu-se que a mesma pode não ser a causa direta do atual processo erosivo em Massaguaçú, uma vez que houve predominância de vulnerabilidades baixas mesmo em anos em que a ocupação esteve crescente. O método utilizado no estudo se mostrou eficiente para responder as questóes propostas; é um procedimento rápido e de baixo custo para a avaliação do ambiente, tornando-o acessível para os gestores e tomadores de decisôes realizarem avaliaçôes rápidas do meio.

Palavras-chave: Indicadores de vulnerabilidade, Sistemas de Informação Geográfica (SIG), planejamento urbano.

\section{ABSTRACT}

Coastal erosion can cause major environmental and social damage to coastal areas, and disorderly and inadequate human occupation at these sites can lead to erosion.

Massaguaçu Beach, on the northern coast of São Paulo State, has suffered severe erosion in recent years. In the most severe cases, erosion had already begun to affect the road way Dr. Manuel Hyppolito Rego (SP-55) that runs next to the beach. Several efforts have been implemented to contain this problem, but have proved to be unsuccessful. The cause of these erosions remain unclear, however, studies suggest that these changes in the coast line may be related to the increasing urbanization around the beach.

Because of this phenomenon, this study aimed to analyze the coastal erosion vulnerability at the beach over a period of 40 years, with the objective of evaluating if human occupation had a significant impact in the local erosion. Data was gathered using nine vulnerability indicators: beach width, shoreline position, offshore settings, presence of rivers andlor inlets, terrain elevation, vegetation, coastal engineering structures, occupation rate and soil permeability.

@ - Corresponding author: juliana.santos.ribeiro@usp.br, jusantosribeiro@gmail.com

1 - Instituto Oceanográfico - Universidade de São Paulo, Praça do Oceanográfico, 191. São Paulo, Brasil

* Submission: 29 October 2012; Evaluation: 4 December 2012; Revised manuscript: ...; Accepted: 4 December 2012; Available on-line: 31 May 2013 
The methodology consisted of analysis of several aerial pictures of the beach (from 1962, 1973, 1977, 1987 e 2001) and data from earlier works. To facilitate the analysis of Massaguaçú beach, its $7.5 \mathrm{~km}$ stretch was divided into three distinct sectors: north, center, and south sector. The division was made based on the different features present in each sector. The north sector is characterized by the presence of the Cocanha Islands, which serve as a natural barrier for the beach, and the Bracui river. The center sector contains the largest stretch of the road way SP-55 that runs next to the entirety of the center sector, and has absence of rivers and offshore barriers. The south sector is characterized by the absence of the road way and by the presence of the Capricórnio River.

The results show that coastal erosion vulnerability has increased in a non-constant way over the analyzed period. The indicators beach width and shoreline position demonstrated oscillating behavior, which in turn made the final vulnerability result oscillate as well. The other indicators demonstrated stable or increasing vulnerability results. The central part of Massaguaçu-where erosion is intense-presented the highest rates of coastal erosion vulnerability over time.

Although human occupation seemingly has been influential in the results, the study concluded that it might not be the direct cause of the current erosion in Massaguaçu. This holds true because there was a predominance of low vulnerability even in years where the occupation was growing. Recent studies suggested that the largest contribution to the recording of the erosion in the studied area came from the longshore current that transports sediment from the center sector to the neighboring sectors.

The method used in this study has proven effective in answering the questions posed. It is a quick, inexpensive method to evaluate the environment, making it accessible for coastal managers to carry out rapid environmental assessments. As a result, better understanding of areas vulnerable to erosion may lead to more responsible city planning along the coastal area.

Keywords: Indicators of vulnerability, Geographic Information System (GIS), urban planning.

\section{INTRODUÇÁO}

\subsection{Motivaçáo}

A avaliação da vulnerabilidade à erosão costeira tem sido uma ferramenta importante para identificar e prevenir impactos socioeconômicos nas cidades litorâneas. Entretanto, a lacuna oriunda da escassez de dados de monitoramento de longo prazo da zona costeira, sobretudo no Brasil, é um obstáculo difícil de ser vencido. O esforço realizado nesse sentido tem partido de pesquisadores com interesse fundamentalmente científico.

Ao longo de praticamente todo o litoral brasileiro são verificados pontos de erosão costeira, resultantes de processos que, em muitos casos, são fruto de açóes humanas que causam interferências na morfodinâmica e no aporte sedimentar (Muehe, 2005; 2006). Muehe (2005) não considera esses processos erosivos como uma ameaça para a totalidade da orla, contudo esses processos têm se traduzido em danos socioeconômicos, ambientais e culturais significativos na medida em que casas são instaladas na póspraia, a infraestrutura urbana é afetada e obras de engenharia costeira são construídas.

De forma sumarizada, segundo Cai et al. (2009), as causas da erosão costeira de escala global ou regional podem ser naturais: aumento do nível do mar, intensificaçáo de tempestades, subsidência tectônica, alteraçóes nas bacias hidrográficas; e antrópicas: subsidência do solo e construção de barragens. À escala local, podem-se considerar relevantes as construçôes que interferem na deriva litoral, e a retirada de areia no sistema fluvial-litoral. Os processos naturais e antrópicos que resultam na erosão costeira inter-relacionamse ao reduzir o balanço sedimentar ou alterar a dinâmica costeira. Além da perda de área emersa, outros impactos são, sobretudo, a intrusão marinha nas águas superficiais, a curto prazo, e a intrusão de água salgada no lençol freático e a perda da biodiversidade a longo prazo (Nicholls 2004; Nicholls \& Cazenave, 2010).

Nesse contexto, a projeção de aumento do nível do mar
(NM) feita pelo IPCC (2007) para 2100 foi de 0,2 a 0,6 m. Adicionalmente, existem projeçóes como a de Rahmstorf (2007) onde o cenário mais otimista para o mesmo período é de um aumento da ordem de $0,6 \mathrm{~m}$ e o mais pessimista é de 1,4 $\mathrm{m}$. Esses estudos são importantes para fins de planejamento e adaptação. No caso de algumas praias onde um rápido aumento do NM foi observado, foi constatado que as açóes de adaptaçáo partem primeiramente da população afetada e pouco ou quase nada é feito pelo governo (Linnekamp et al., 2011).

Certamente as açóes com vista à adaptação ao aumento do NM afetam diretamente a vida da população que habita as proximidades do litoral. No caso do delta de Ebro, Espanha, Fatorić \& Chelleri (2012) constataram que a comunidade mostrou preocupação em relação a como a adaptação seria feita. Esta optou por um processo suave, com ação realizada em consonância com a manutenção dos recursos naturais da região.

Sabe-se, no entanto, que os impactos humanos podem somar-se àqueles causados pela natureza. Além disso, sobretudo às escalas local e regional, podem ser mais danosos do que processos naturais que atuam em grande escala temporal e espacial, como as mudanças climáticas. A melhor forma de lidar com prejuízos causados por processos erosivos é tomar medidas preventivas antes que eles atinjam níveis mais críticos. Desta maneira, estudos que contemplem a avaliação da vulnerabilidade à erosão costeira assumem papel primordial para o planejamento urbano e regional em áreas litorâneas.

A vulnerabilidade da zona costeira pode ser avaliada considerando diferentes fatores, como o aumento do nível do mar, erosão costeira e tempestades. Vários trabalhos foram feitos considerando esses fatores (Pendleton et al., 2010; Bush et al., 1999; Bosom \& Jiménez, 2011), no entanto, todos foram realizados para áreas de grande extensão geográfica e utilizando bancos de dados com vários anos de monitoramento. Alguns estudos de vulnerabilidade costeira já foram realizados na praia de Massaguaçú (SP) (Figura 1) 
(Sousa et al., 2011; Sousa et al., 2013). Entretanto, esses trabalhos fizeram uma análise instantânea da vulnerabilidade com base em informaçóes recentes. Diferentemente, o objetivo deste trabalho é avaliar a evolução da vulnerabilidade à erosão costeira local ao longo de 40 anos observando como se deu o crescimento urbano e como este influenciou os processos erosivos atuais na região. $\mathrm{O}$ estudo tem como base uma série de indicadores de ordem, sobretudo, qualitativa, adaptados para a área em apreço: largura da praia, posição da linha de costa, configuraçóes ao largo, presença de rios e/ou desembocaduras, elevação do terreno, vegetação, obras de engenharia costeira, taxa de ocupação e permeabilidade do solo. Através destes, foram estabelecidos graus de vulnerabilidade nos diferentes anos e setores do arco praial.

\section{2. Área de estudo}

A praia de Massaguaçú (Figura 1), pertencente ao município de Caraguatatuba (litoral norte de São Paulo), está localizada próximo ao Trópico de Capricórnio, possui $7,5 \mathrm{~km}$ de extensão e orientação NE-SE. A praia é composta por areia grossa e média, com grau de selecionamento moderado (Rogacheski, 2010). A maior parte da praia apresenta morfologia com características de praia refletiva de alta energia, com tendências intermediárias (Sousa \& Luna, 2010). A linha praial é interrompida por dois pequenos rios que alcançam o oceano nas extremidades da praia: rio Capricórnio ao sul e rio Bracuí ao norte.

A área está sujeita a um regime de micro-marés (Sousa et al., 2013). Quanto ao clima de ondas, a direção dominante da onda é leste no verão (com período de 6-8 s e altura de onda de 1-2 m) e sul nas demais estaçóes (com períodos de 10-12 s e alturas de onda de $1-2 \mathrm{~m}, 1-3 \mathrm{~m}$ e $2-3 \mathrm{~m}$ para verão, outono e inverno, respectivamente), de acordo com os resultados de Pianca et al. (2010) para a costa sudeste brasileira.

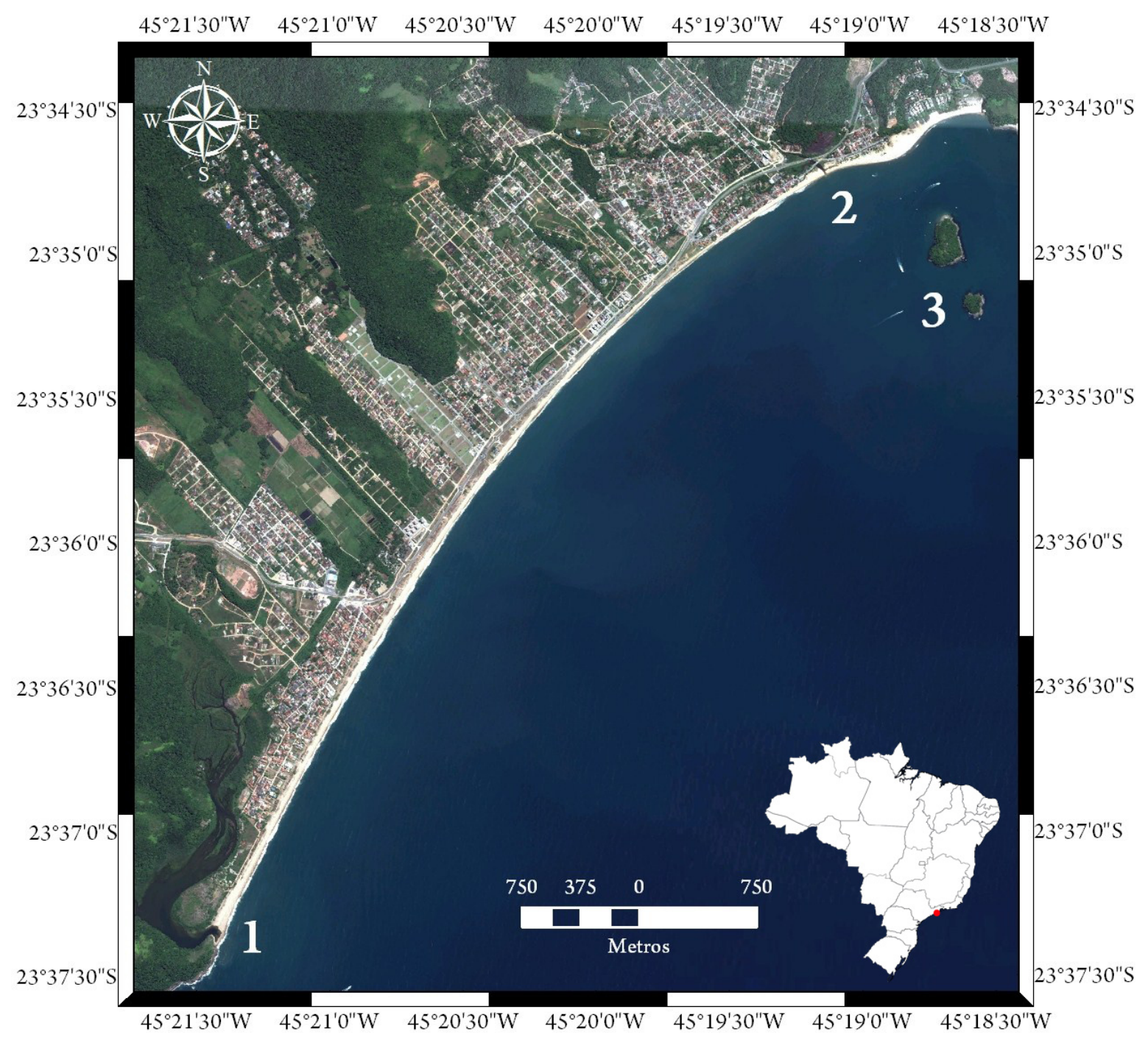

Figura 1. Mapa da área de estudo, onde 1 representa a desembocadura do Rio Capricórnio, 2 representa a desembocadura do Rio Bracuí e 3 representa as Ilhas Cocanha.

Figure 1. Map of the study area, where 1 represents the Capricórnio River inlet, 2 represents the Bracui River inlet and 3 represents the Cocanha Islands. 


\section{MATERIAL E MÉTODOS}

Foram utilizadas técnicas de geoprocessamento baseadas em Sistemas de Informaçôes Geográficas (SIG). Para isso, a praia de Massaguaçú, com extensão de $7,5 \mathrm{~km}$, foi dividida em três setores: norte, centro e sul. As técnicas utilizadas para este trabalho estáo descritas a seguir.

\subsection{Imagens aéreas}

A maioria dos indicadores de vulnerabilidade utilizados para estimar a evolução da vulnerabilidade à erosão foram analisados com base em imagens digitais. Para tanto, utilizaram-se conjuntos de imagens aéreas abrangendo um período de aproximadamente 40 anos (Tabela 1).

As imagens foram utilizadas para determinar o grau de vulnerabilidade da área em cada ano e gerar mapas temáticos para uma melhor visualização dos indicadores analisados. O material foi fornecido pelo Instituto Oceanográfico da Universidade de São Paulo (fotografias aéreas dos anos de 1962, 1973, 1977 e 1987) e pelo INPE (Instituto Nacional de Pesquisas Espaciais; fotografia digital do ano de 2001).

A imagem de 2001 foi fornecida georreferenciada no sistema de coordenadas UTM (Universal Transverse Mercator), fuso $23 \mathrm{~S}$ e datum planimétrico SAD69 (South American Datum 1969). Desta forma, essa imagem serviu como base para o georreferenciamento das demais que foram projetadas através dos programas IDRISI 15.0 - Andes Edition e ArcMAP 9.3 da ESRI (Environmental Systems Research Institute).

Para georreferenciar as imagens a partir da imagem de 2001, utilizaram-se pontos de controle (PCs), que são locais identificados tanto na imagem base quanto na imagem a ser georreferenciada. Os PCs devem ser distribuídos ao longo da área de interesse para que se obtenha um melhor desempenho no processo (Araujo et al., 2009); no presente estudo, priorizou-se a precisão do georreferenciamento na linha de costa das fotografias.
Durante o processo, o erro encontrado no georreferenciamento (RMS - Root Mean Square) foi sempre menor que o erro máximo permitido, que corresponde ao erro de graficismo da imagem (Júnior et al., 2006). A resolução das imagens após digitalização foi de 300 DPIs.

\subsection{Delimitaçáo dos setores e extensão da faixa costeira analisada}

Para facilitar a análise, o arco praial de Massaguaçú, com extensão de $7,5 \mathrm{~km}$, foi dividido em três setores distintos: setor norte, setor centro e setor sul. Essa divisão se deu em virtude das diferentes feiçôes encontradas ao longo da área de estudo (Figura 1). No setor norte há a presença das Ilhas Cocanha, que servem de barreira natural para a praia, além da presença do rio Bracuí. O setor centro é caracterizado por conter a maior extensão da rodovia, sendo que esta margeia a praia por todo o comprimento do setor. Além disso, nessa área há ausência de rios e barreiras naturais ao largo. Já o setor sul é caracterizado pela quase ausência da rodovia, e pela presença do rio Capricórnio. A extensão da faixa costeira analisada foi de $500 \mathrm{~m}$, partindo do limite da linha d'água (obtida através da imagem do ano de 2001) em direção ao interior.

\subsection{Indicadores de Vulnerabilidade}

Para se obter o grau de vulnerabilidade à erosão da área de estudo, nove indicadores foram analisados. Foram utilizados parâmetros qualitativos e quantitativos para classificar os indicadores como sendo de baixa, média ou alta vulnerabilidade (Tabela 2).

Os indicadores analisados foram adaptados de Sousa et al. (2013) e são brevemente descritos abaixo:

Posição da linha de costa: linhas de costa estáveis ou em progradação representam um cenário de baixa vulnerabilidade, enquanto que linhas de costa que sofrem retrogradação evidenciam um cenário de alta vulnerabilidade.

Tabela 1. Detalhamento das imagens/fotografias aéreas utilizadas no estudo.

Table 1. Details of the aerial images/photographs used in this study.

\begin{tabular}{|c|c|c|c|c|c|}
\hline Ano & Mês & $\begin{array}{l}\text { Tipo de } \\
\text { material }\end{array}$ & $\begin{array}{l}\text { Número das } \\
\text { fotografias/ } \\
\text { imagens aéreas }\end{array}$ & $\begin{array}{l}\text { Escala / } \\
\text { resoluçãoo }\end{array}$ & $\begin{array}{c}\text { Entidade / } \\
\text { empresa responsável }\end{array}$ \\
\hline 1962 & Outubro & Fotografias aéreas & $\begin{array}{l}\text { 7-9280, 7-9536, } \\
\text { 7-9538 }\end{array}$ & $1: 25.000$ & $\begin{array}{l}\text { Secretaria de Agricultura do Estado de } \\
\text { São Paulo - Instituto Agronômico (AS - } \\
\text { IA)/ Aerofoto Natividade S.A. }\end{array}$ \\
\hline 1973 & Janeiro & Fotografias aéreas & 43183,43208 e 43210 & $1: 25.000$ & $\begin{array}{c}\text { Base Service (Base Serviços de Fotografias } \\
\text { Aéreas e Imagens LTDA) }\end{array}$ \\
\hline 1977 & $\begin{array}{l}\text { Não foi } \\
\text { informado }\end{array}$ & Fotografia aérea & 1928 & $1: 35.000$ & $\begin{array}{c}\text { Base Service (Base Serviços de Fotografias } \\
\text { Aéreas e Imagens LTDA) }\end{array}$ \\
\hline 1987 & Janeiro & Fotografias aéreas & $\begin{array}{l}0009,0011,0014 \\
0016,0014,0016\end{array}$ & $1: 10.000$ & $\begin{array}{c}\text { Base Service (Base Serviços de Fotografias } \\
\text { Aéreas e Imagens LTDA) }\end{array}$ \\
\hline 2001 & $\begin{array}{l}\text { Não foi } \\
\text { informado }\end{array}$ & Imagem aérea & $2796-12$ & 1 metro & $\begin{array}{c}\text { INPE (Instituto Nacional de Pesquisas } \\
\text { Espaciais) }\end{array}$ \\
\hline
\end{tabular}


Tabela 2. Descrição dos indicadores de vulnerabilidade utilizados no presente trabalho. A vulnerabilidade mencionada é a vulnerabilidade à erosão costeira (adaptado de Sousa et al., 2013).

Table 2. Description of the indicators of vulnerability used in this study. The vulnerability mentioned is the erosion vulnerability (adapted from Sousa et al., 2013).

\begin{tabular}{|c|c|c|c|}
\hline $\begin{array}{c}\text { Indicador de } \\
\text { vulnerabilidade }\end{array}$ & Vulnerabilidade baixa & Vulnerabilidade moderada & Vulnerabilidade alta \\
\hline $\begin{array}{c}\text { Posição da } \\
\text { linha de costa }\end{array}$ & Progradação & Estável & Retrogradação \\
\hline Largura da praia & Larga faixa de praia & Largura média a estreita & Praia estreita \\
\hline $\begin{array}{l}\text { Presença de rios e / ou } \\
\text { desembocaduras }\end{array}$ & Distância maior que $100 \mathrm{~m}$ & Distância entre 50 e $100 \mathrm{~m}$ & Distância menor que $50 \mathrm{~m}$ \\
\hline Elevação do terreno & Maior que $6 \mathrm{~m}$ & Entre 3 e $6 \mathrm{~m}$ & Menor que $3 \mathrm{~m}$ \\
\hline Taxa de ocupação & Menor que $30 \%$ & Entre 30 e $70 \%$ & Maior que $70 \%$ \\
\hline $\begin{array}{l}\text { Permeabilidade } \\
\quad \text { do solo }\end{array}$ & $\begin{array}{l}\text { Permeável, com nenhuma } \\
\text { ou pouca ocupaçáo }\end{array}$ & $\begin{array}{l}\text { Moderadamente permeável, } \\
\text { em função da ocupação/ } \\
\text { urbanização }\end{array}$ & $\begin{array}{c}\text { Permeabilidade seriamente } \\
\text { afetada, com presença de } \\
\text { ocupaçáo urbana bem } \\
\text { desenvolvida }\end{array}$ \\
\hline $\begin{array}{l}\text { Configuraçóes } \\
\text { ao largo }\end{array}$ & $\begin{array}{l}\text { Presença de barreiras } \\
\text { naturais (ilhas, recifes ou } \\
\text { rochas de praia) }\end{array}$ & $\begin{array}{c}\text { Pista de atuação do vento } \\
\text { limitada (presença de barreiras } \\
\text { arenosas ao largo) }\end{array}$ & $\begin{array}{l}\text { Pista de atuação do vento } \\
\text { extensa, sem obstáculos naturais } \\
\text { que minimizem a energia das } \\
\text { ondas }\end{array}$ \\
\hline Vegetação & $\begin{array}{l}\text { Densa com florestas } \\
\text { desenvolvidas e sem } \\
\text { evidências de erosão }\end{array}$ & $\begin{array}{c}\text { Bem estabelecida, com gramíneas } \\
\text { e arbustos }\end{array}$ & Nenhuma ou pouca vegetação \\
\hline $\begin{array}{c}\text { Obras de } \\
\text { engenharia costeira }\end{array}$ & $\begin{array}{l}\text { Ausência de estruturas } \\
\text { costeiras }\end{array}$ & $\begin{array}{l}\text { Estruturas pequenas ou pouco } \\
\text { significantes }\end{array}$ & $\begin{array}{l}\text { Presença de quebra-mares, } \\
\text { esporóes, molhes, etc. }\end{array}$ \\
\hline
\end{tabular}

Largura da praia (ao longo do arco praial): sistemas praiais largos são mais propensos à estabilidade.

Configuraçóes ao largo: a área de mar aberto que se encontra adjacente à linha de costa determina, parcialmente, a altura das ondas (de tempestade ou não) que podem surgir. Este indicador tem relação com feições migratórias ou fixas que, em geral, funcionam como barreiras que atenuam o ataque das ondas à zona costeira.

Obras de engenharia costeira: as obras podem indicar instabilidade atual ou recente da linha de costa, uma vez que o objetivo delas é conter a erosão costeira ou proteger construções presentes no local, mas também tendem a induzir erosão a sotamar.

Elevação do terreno: a elevação do sistema praia-duna e póspraia é um fator primordial quando se trata da suscetibilidade à inundação. Áreas baixas e planas estão expostas a ataques de ondas, sobrelavagem, inundação por tempestade (incluindo a ondulação e a sobreelevação metereológica), e às vezes transporte eólico de areia.

Vegetação: a presença de vegetação bem desenvolvida, seja ela rasteira, arbustiva ou arbórea na zona de pós-praia, sugere um cenário de baixa erosão e rara intrusão de água salina.
Presença de rios elou desembocaduras: essas feições podem migrar por centenas de metros, sugerindo instabilidade em suas adjacências.

Permeabilidade do solo: a permeabilidade dos solos está intimamente relacionada com a ocupação e com o tipo de uso do solo. Solos pouco permeáveis são mais susceptíveis à inundação.

Taxa de ocupação: a ocupação da zona costeira altera o ambiente natural, seja com o desmatamento de vegetação nativa, com a impermeabilização do solo, a criação de corredores de penetração de inundaçóes ou com outras ações. Ademais, as estruturas construídas são muito afetadas por processos erosivos. Quanto maior a taxa de ocupação do local, maior a vulnerabilidade à erosão costeira.

\subsubsection{Posiçấo da linha de costa}

A linha de costa (LC), do ponto de vista físico, corresponde ao ponto de encontro entre o continente e o oceano. Deve-se entender que a LC é uma linha móvel, em escalas espacial e temporal, devido aos inúmeros fatores que atuam nesse ambiente dinâmico (processos erosivos e deposicionais, marés, ondas, dentre outros; Araujo et al., 2009). Tendo em 
vista essa mobilidade, é importante que haja a definição de um critério para delimitação da LC, para que os resultados obtidos possam ser comparados.

Sáo diversos os indicadores que podem ser utilizados para se delimitar a linha de costa, como o limite da vegetação costeira, a linha d'água e a interface da zona entremarés (correspondente ao limite máximo da preamar). Adelimitação deve ser feita pelo indicador mais discernível e constante no material analisado (Araujo et al., 2009; Boak \& Turner, 2005), que no caso das imagens aéreas utilizadas no presente estudo é a interface água - sedimento. $\mathrm{Na}$ área de estudo, em função de mudanças na linha de vegetação causadas pela ação antrópica (plantio nas margens da estrada), optou-se pela utilização da linha d'água como indicador de linha de costa. Apesar das possíveis limitaçóes envolvidas na análise desse parâmetro, ele apresentou-se como melhor opção para a área de interesse.

As linhas de costa foram digitalizadas com o uso do ArcGIS 9.3. Para determinar a variação dessas linhas nos vários anos, uma rotina em ambiente MATLAB foi criada. A função consiste em analisar a variaçáo de duas linhas de costa a partir de "n" transectos traçados perpendicularmente a uma linha de base suavizada. O retorno da função é uma matriz de duas colunas, sendo a primeira a coordenada do meridiano UTM do transecto e a segunda um valor positivo ou negativo representando a distância de progradação ou retrogradaçáo da costa, respectivamente.

Para o presente estudo, foram utilizados 150 transectos ao longo da praia, com espaçamento de $50 \mathrm{~m}$. Os transectos foram traçados a partir da linha de costa do ano de 2001.

\subsubsection{Largura da praia}

A partir da imagem aérea de 2001, foram traçados 37 transectos ao longo da área de estudo, perpendiculares à linha de costa e distantes um dos outros cerca de 200 metros. A largura da praia de cada ano corresponde à medida do comprimento do segmento de cada transecto que tinha como limites a linha d'água e a faixa de vegetação. Para determinar o grau de vulnerabilidade de cada setor, fezse uma divisão do comprimento dos transectos em três intervalos iguais, considerando como extremos o maior e menor valor encontrado ao longo dos anos. Assim, os setores que apresentaram um maior número de transectos estreitos (de 8,78 a 37,17 metros) foram classificados como de alta vulnerabilidade, os que apresentaram transectos de média extensão (37,18 a 65,56 metros) foram considerados de média vulnerabilidade e os setores que continham em sua maioria transectos largos (65,57 a 93,96 metros) foram classificados como sendo de baixa vulnerabilidade.

\subsubsection{Presença de rios e / ou desembocaduras}

A vulnerabilidade quanto a esse indicador foi analisada a partir da distância de cada setor aos rios presentes na praia. Nos setores em que havia a presença de rios ou desembocaduras, a distância considerada foi zero (alta vulnerabilidade). Nos outros setores, a vulnerabilidade foi considerada baixa se a distância dos rios era maior que 100 metros e considerada média se a distância era entre 50 e 100 metros. Para esse cálculo, foram traçados polígonos em volta dos rios que tivessem uma distância fixa dos mesmos (função buffer do ArcGIS 9.3), criando "áreas de influência" dos rios de 50 e 100 metros. A partir disso, pôde-se ter certeza do quão distante cada setor estava dos rios.

\subsubsection{Elevação do terreno}

Em virtude da inexistência de dados para a região costeira na praia de Massaguaçú, foram utilizados dados de altitude extraídos do projeto "TOPODATA - Banco de Dados Geomorfométricos do Brasil” (INPE, 2008). A partir das curvas de nível obtidas, criou-se uma rede triangular irregular (TIN - Triangular Irregular Network) para facilitar a análise altimétrica. O método de interpolação empregado foi a triangulação de Delaunay.

A TIN foi dividida em três intervalos de altura: menor que 3 metros (alta vulnerabilidade), entre 3 e 6 metros (média vulnerabilidade) e maior que 6 metros (baixavulnerabilidade). A partir desse mapa, foram traçados polígonos ao redor das regióes de mesma altura, e suas áreas foram calculadas para se obter a vulnerabilidade predominante em cada setor.

Como as imagens aéreas antigas não possuem informaçóes altimétricas e o período analisado é muito curto para se considerar variações expressivas da elevação do terreno, considerou-se a mesma altura para todas as fotografias aéreas analisadas.

\subsubsection{Taxa de ocupação}

Os indícios de ocupação humana foram vetorizados em cada conjunto de fotografias aéreas, através da criação de polígonos. Calculou-se, então, a área desses polígonos, para se obter a área correspondente à ocupação. Habitaçóes foram consideradas como sendo ocupação, assim como a rodovia SP-55, que é pavimentada. Lotes vazios não foram considerados como ocupação.

A taxa de ocupação em porcentagem (T) foi obtida, para cada ano e setor analisados, através da Equação 1:

$$
T=\frac{A_{o}}{A_{u}} \times 100
$$

onde $\mathrm{A}_{\mathrm{o}}$ representa a área de ocupação e $\mathrm{A}_{\mathrm{u}}$ representa a área útil.

A área útil $\left(A_{u}\right.$, representando aqui a área passível de ocupação) foi determinada a partir da Equação 2:

$$
A_{u}=A_{c}-A_{r}
$$

onde A representa a totalidade da área continental do segmento analisado e $\mathrm{A}_{\mathrm{r}}$ representa a área ocupada pelos rios.

É importante ressaltar que a área útil dos setores foi diferente para cada ano, uma vez que o curso dos rios e a posição da linha de costa mudaram durante o período estudado. 


\subsubsection{Permeabilidade do solo}

A permeabilidade do solo foi classificada de acordo com o resultado da taxa de ocupaçáo, uma vez que ela é relativa à pavimentação e à urbanização do local analisado. Assim, nos setores que apresentaram alta taxa de ocupação o indicador foi considerado como de alta vulnerabilidade, para taxa média de ocupação o indicador foi considerado de média vulnerabilidade e para baixa taxa de ocupação, baixa vulnerabilidade.

\subsubsection{Demais indicadores}

Os indicadores configuraçóes ao largo, vegetação e obras de engenharia costeira (vide Tabela 2) foram classificados a partir de dados da literatura e observação visual das fotografias aéreas.

\section{RESULTADOS}

\subsection{Indicadores de vulnerabilidade}

Os indicadores de vulnerabilidade foram analisados e classificados de acordo com sua respectiva vulnerabilidade (alta, média ou baixa) para cada ano analisado. A compilação dos resultados da análise da vulnerabilidade dos indicadores está apresentada na Tabela 3, e alguns dos resultados estáo descritos em detalhes a seguir.

\subsubsection{Posiçáo da linha de costa}

Através da função de MATLAB já explicitada no item 2.1.1 determinou-se se a linha de costa progradou ou retrogradou nos intervalos de tempo especificados. A rotina gerou gráficos com informaçôes quantitativas da variação da linha de costa. Quando os resultados apresentaram um maior número de perfis em progradação, a vulnerabilidade correspondente ao setor foi baixa. Já nos setores em que os resultados eram de retrogradaçáo em sua maioria, a vulnerabilidade foi alta. O ano de 1962 náo apresentou resultados para esse indicador porque não havia linha de costa anterior para se comparar.

A Figura 2 apresenta os gráficos da variaçáo da posição da LC entre os anos subsequentes e setores analisados. O setor norte apresentou retrogradaçáo no período de 1962 a 1977 e progradação no período de 1977 a 2001. O setor centro apresentou progradação nos períodos de 1973 a 1987

Tabela 3. Vulnerabilidades encontradas para cada indicador analisado, onde "N", "C" e "S" representam setor norte, centro e sul, e "A", "M" e "B" representam alta, média e baixa vulnerabilidade, respectivamente.

Table 3. Results of vulnerability for each analyzed indicator, where " $N$ ", " $C$ " and " $S$ " represent north, centre and south sectors accordingly, and " $A$ ", " $M$ " and " $B$ " represent high, moderate and low vulnerability, respectively.

\begin{tabular}{|c|c|c|c|c|c|c|c|c|c|c|c|c|c|c|c|}
\hline \multirow{2}{*}{$\begin{array}{c}\text { Indicador de } \\
\text { vulnerabilidade }\end{array}$} & \multicolumn{3}{|c|}{1962} & \multicolumn{3}{|c|}{1973} & \multicolumn{3}{|c|}{1977} & \multicolumn{3}{|c|}{1987} & \multicolumn{3}{|c|}{2001} \\
\hline & $\mathrm{N}$ & $\mathrm{C}$ & S & $\mathrm{N}$ & $\mathrm{C}$ & S & $\mathrm{N}$ & $\mathrm{C}$ & S & $\mathrm{N}$ & $\mathrm{C}$ & S & $\mathrm{N}$ & $\mathrm{C}$ & S \\
\hline $\begin{array}{l}\text { Posição da } \\
\text { linha de costa }\end{array}$ & - & - & - & A & A & A & A & B & A & B & B & B & B & A & B \\
\hline Largura da praia & M & M & M & A & A & A & A & A & A & A & M & A & M & A & M \\
\hline $\begin{array}{l}\text { Presença de rios e/ou } \\
\text { desembocaduras }\end{array}$ & A & B & A & A & B & A & A & B & A & A & B & A & A & B & A \\
\hline Elevação do terreno & M & M & M & M & M & M & M & M & M & M & M & M & M & M & M \\
\hline Taxa de ocupação & B & B & B & B & B & B & B & B & B & B & B & B & M & M & M \\
\hline $\begin{array}{l}\text { Permeabilidade } \\
\text { do solo }\end{array}$ & B & B & B & B & B & B & B & B & B & B & B & B & M & M & M \\
\hline $\begin{array}{l}\text { Configuraçóes } \\
\text { ao largo }\end{array}$ & B & A & A & B & A & A & B & A & A & B & A & A & B & A & A \\
\hline Vegetação & B & B & B & M & M & M & M & M & A & A & A & A & A & A & A \\
\hline $\begin{array}{c}\text { Obras de } \\
\text { engenharia costeira }\end{array}$ & B & B & B & B & B & B & B & B & B & B & B & B & B & B & B \\
\hline
\end{tabular}


e retrogradação nos períodos de 1962 a 1973 e 1987 a 2001. Já o setor sul apresentou retrogradação no período de 1962 a 1977 e progradação no período de 1987 a 2001 . Analisando os quase 40 anos como um todo, ou seja, o intervalo entre 1962 e 2001, observa-se que houve progradação no setor norte e retrogradação nos setores centro e sul.

\subsubsection{Largura da praia}

Na medição da extensão praial ao longo da área de estudo, o maior comprimento obtido dentre todos os anos foi 93,96 metros, e o menor foi 8,78 metros. Com a divisão dos resultados em três intervalos iguais, ficou estabelecido que perfis praiais de 8,78 a 37,17 metros seriam considerados de alto risco, de 37,18 a 65,56 metros seriam considerados de médio risco e de 65,57 a 93,96 metros seriam considerados de baixo risco. Os resultados da classificação dos transectos quanto à vulnerabilidade estão apresentados na Tabela 4 .

\subsubsection{Presença de rios e / ou desembocaduras}

$\mathrm{O}$ arco praial da área de estudo apresenta dois rios ao longo de seu comprimento (presentes em todas as imagens aéreas analisadas), o rio Bracuí no setor norte e o rio Capricórnio no setor sul. O setor centro dista mais de 100 metros desses rios.
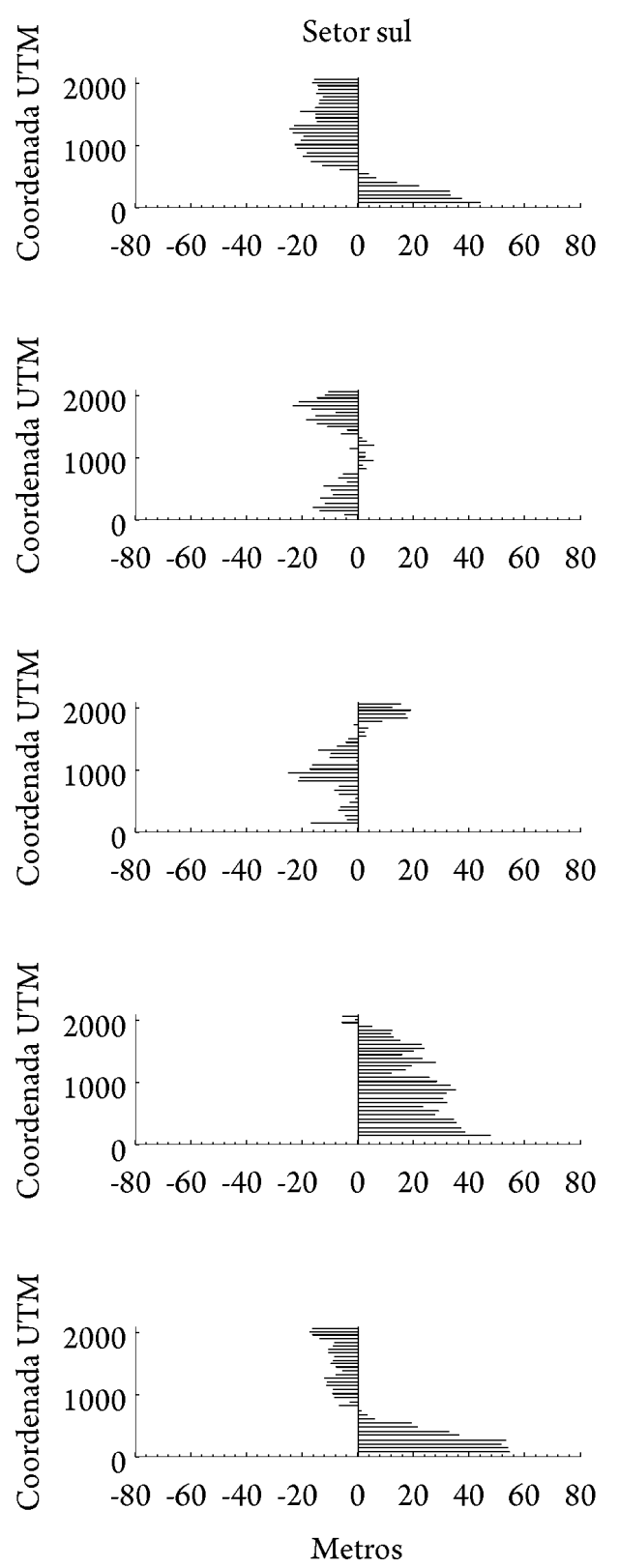

\section{2 a 1973}

Setor centro
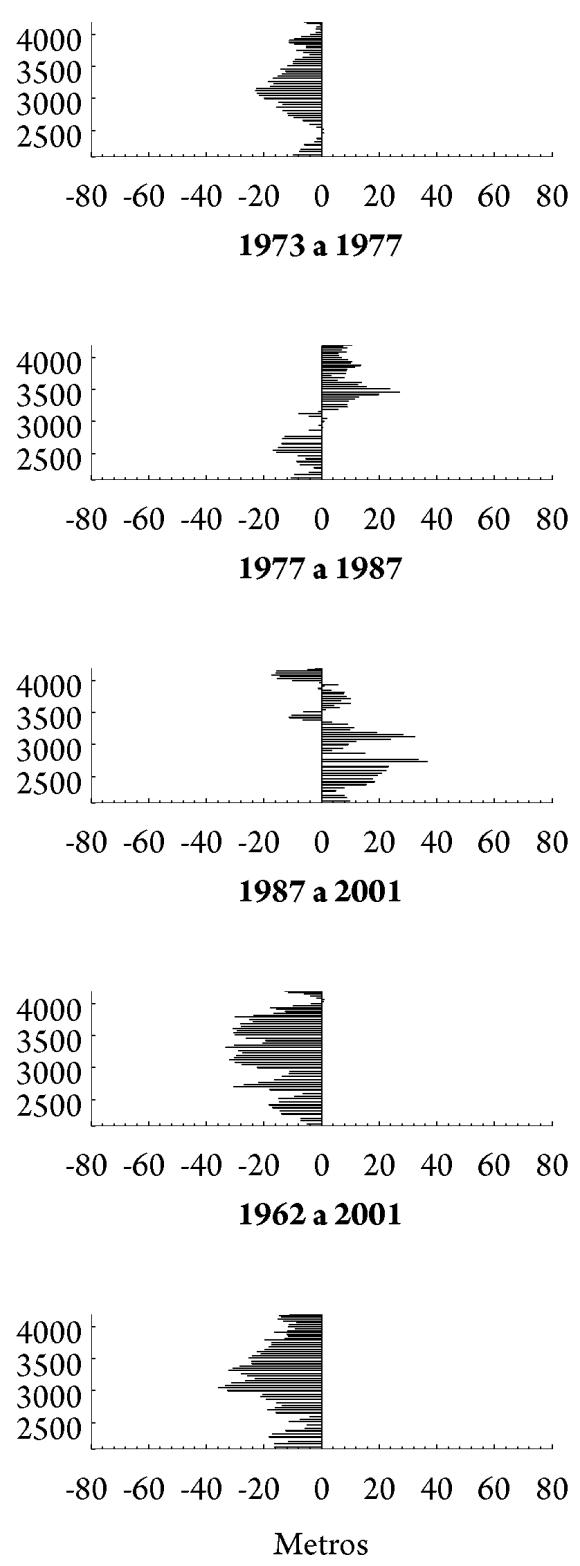

Setor norte
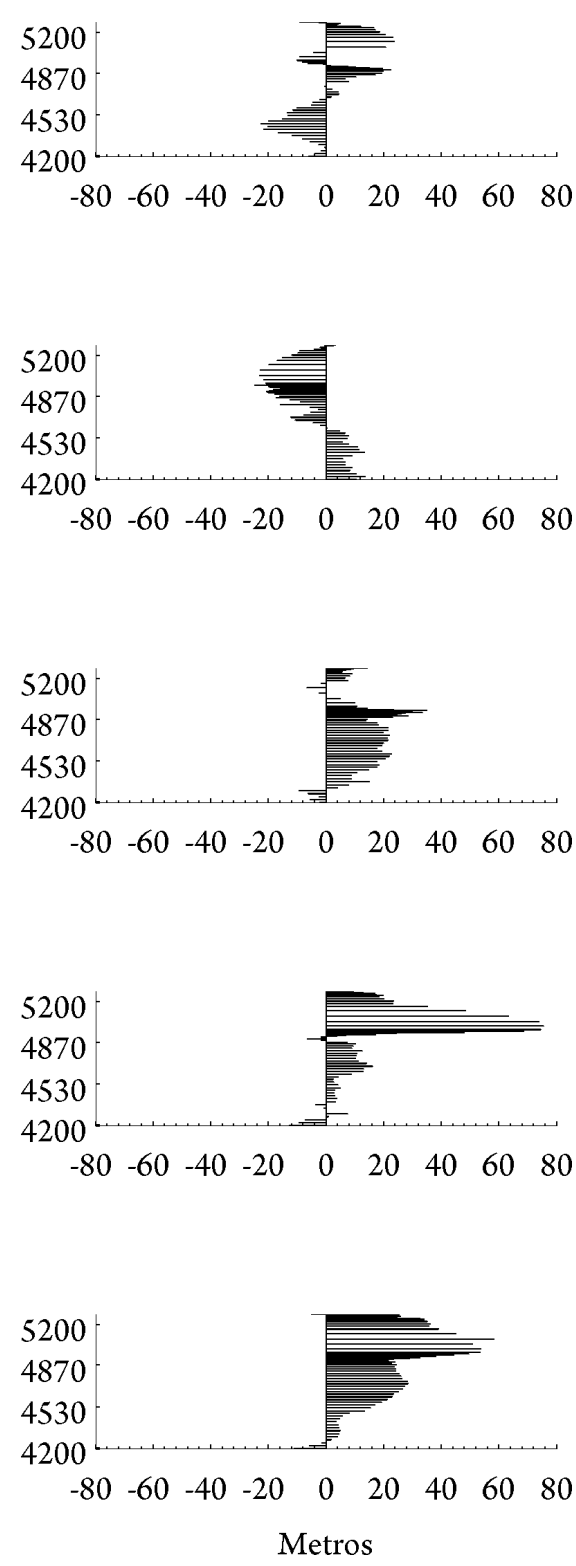

Figura 2. Gráficos das variações de linha de costa nos diferentes anos e setores analisados. Valores positivos indicam progradação e valores negativos indicam retrogradação.

Figure 2. Charts of shoreline variations at the different years and sectors analyzed. Positive values: progradation; negative values: retrogradation. 
Tabela 4. Porcentagem de transectos da largura de praia correspondentes a cada grau de vulnerabilidade, para cada ano analisado.

Table 4. Percentage of beach width lines relative to each vulnerability result for each analyzed year.

\begin{tabular}{|c|c|c|c|c|c|c|c|c|c|c|c|c|c|c|c|}
\hline \multirow{2}{*}{$\begin{array}{c}\text { Grau de } \\
\text { vulnerabilidade }\end{array}$} & \multicolumn{3}{|c|}{1962} & \multicolumn{3}{|c|}{1973} & \multicolumn{3}{|c|}{1977} & \multicolumn{3}{|c|}{1987} & \multicolumn{3}{|c|}{2001} \\
\hline & $\mathrm{N}$ & $\mathrm{C}$ & $S$ & $\mathrm{~N}$ & $\mathrm{C}$ & $S$ & $\mathrm{~N}$ & $\mathrm{C}$ & $S$ & $\mathrm{~N}$ & $\mathrm{C}$ & $S$ & $\mathrm{~N}$ & $\mathrm{C}$ & $S$ \\
\hline Alto & 15 & 7 & 0 & 69 & 100 & 70 & 77 & 64 & 60 & 46 & 36 & 70 & 23 & 100 & 10 \\
\hline Médio & 69 & 86 & 70 & 23 & 0 & 10 & 15 & 36 & 30 & 31 & 64 & 30 & 54 & 0 & 70 \\
\hline Baixo & 15 & 7 & 30 & 8 & 0 & 20 & 8 & 0 & 10 & 23 & 0 & 0 & 23 & 0 & 20 \\
\hline
\end{tabular}

Desse modo, a vulnerabilidade à erosão das imagens aéreas quanto a esse indicador foi classificada como alta nos setores norte e sul e como baixa no setor centro, em todos os anos analisados.

\subsubsection{Elevaçáo do terreno}

A Tabela 5 apresenta as áreas correspondentes a cada tipo de vulnerabilidade, nos diferentes setores. A vulnerabilidade média prevaleceu em todos os setores analisados, representando $47,8 \%, 67,1 \%$ e $44,4 \%$ da área total dos setores norte, centro e sul, respectivamente. Dessa forma, a vulnerabilidade à erosão das imagens aéreas analisadas foi classificada como média em todos os setores e anos analisados em relação a esse indicador.

\subsubsection{Taxa de ocupaçáo}

Ao se analisar a evolução da taxa de ocupação, percebese que a mesma cresceu em todos os setores analisados, especialmente no setor centro, que teve um crescimento praticamente exponencial a partir de 1977 (Figura 3).

A evolução da área ocupada pela urbanização fica ainda mais visível na Figura 4, onde também se pode observar o consequente aumento da impermeabilizaçáo do solo e diminuição da área ocupada pela vegetação. Na figura, a área representada como "continente" representa o solo exposto, aquele não ocupado nem por vegetação nem por ocupação antrópica.

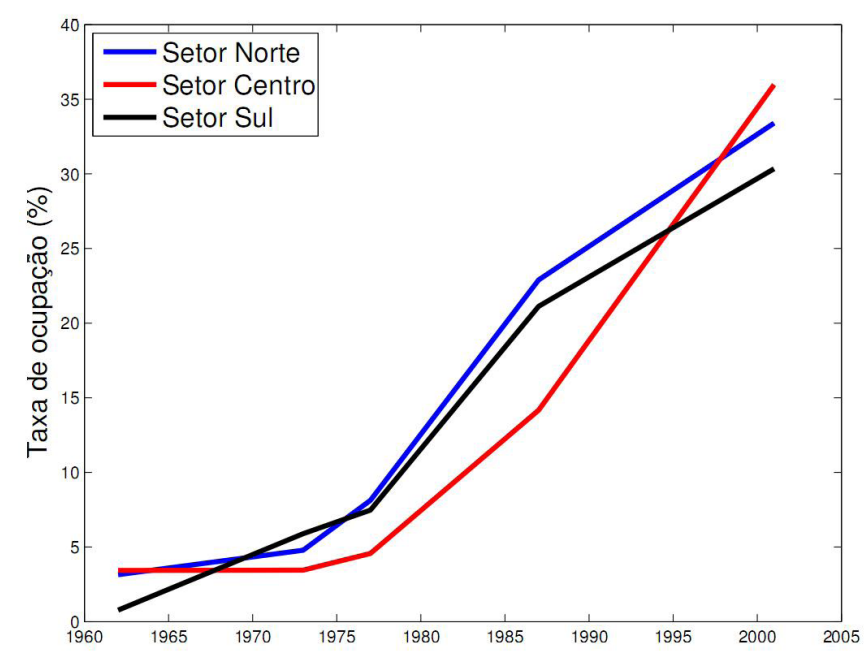

Figura 3. Evolução temporal da taxa de ocupação para cada setor analisado.

Figure 3. Temporal evolution of the occupation rate for each selected sector (blue line: north sector; red line: centre sector; black line: south sector).

Tabela 5. Resultado para a análise de vulnerabilidade quanto à elevação do terreno.

Table 5. Result of the terrain elevation vulnerability analysis.

\begin{tabular}{|c|c|c|c|c|c|c|}
\hline \multirow{2}{*}{$\begin{array}{c}\text { Grau de } \\
\text { vulnerabilidade }\end{array}$} & \multicolumn{2}{|c|}{ Setor norte } & \multicolumn{2}{|c|}{ Setor centro } & \multicolumn{2}{|c|}{ Setor sul } \\
\hline & Área $\left(\mathrm{m}^{2}\right)$ & $\begin{array}{l}\text { Porcentagem } \\
\text { correspondente }\end{array}$ & Área $\left(\mathrm{m}^{2}\right)$ & $\begin{array}{l}\text { Porcentagem } \\
\text { correspondente }\end{array}$ & Área $\left(\mathrm{m}^{2}\right)$ & $\begin{array}{l}\text { Porcentagem } \\
\text { correspondente }\end{array}$ \\
\hline Alta & 130548,8 & 10,6 & 66109,8 & 4,7 & 356290,5 & 27,2 \\
\hline Média & 585180,0 & 47,8 & 951908,5 & 67,1 & 580024,3 & 44,4 \\
\hline Baixa & 508420,0 & 41,5 & 400161,8 & 28,2 & 370994,5 & 28,4 \\
\hline
\end{tabular}




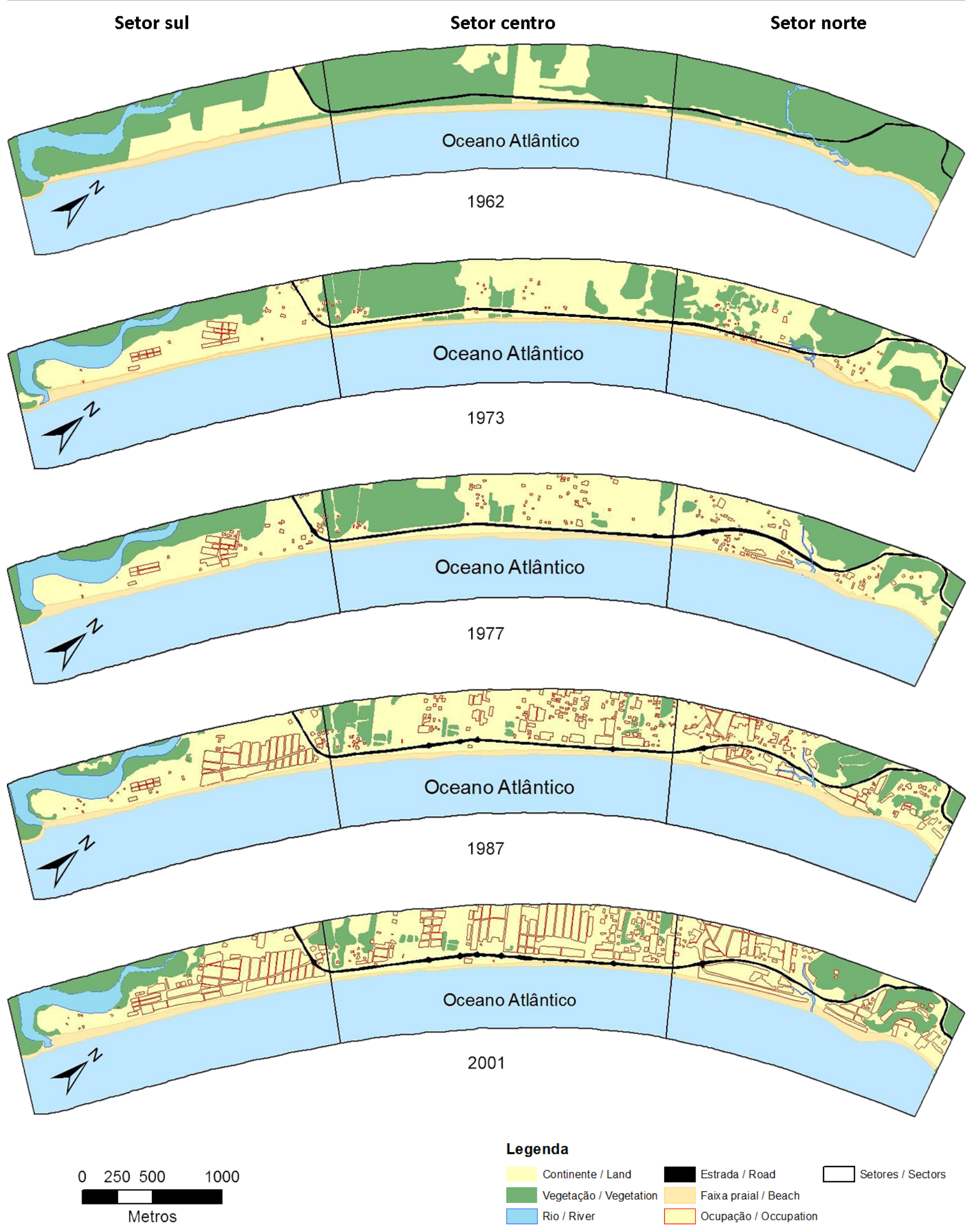

Figura 4. Mapa temático da área de estudo.

Figure 4. Thematic map of the study area. 


\subsubsection{Permeabilidade do solo}

A permeabilidade do solo foi classificada como média no ano de 2001, para todos os setores, e como baixa nos demais anos e setores. Essa classificação seguiu a classificação da taxa de ocupação.

\subsubsection{Configuraçóes ao largo}

No setor norte da praia encontram-se as Ilhas Cocanha, que protegem a costa fazendo com que as ondas incidentes percam energia antes de atingir a praia. Essa configuração caracteriza uma vulnerabilidade baixa.

Já nos setores centro e sul não há presença de barreiras naturais que amenizem o efeito das ondas sobre a costa. Portanto, a vulnerabilidade nesses setores é vista como alta.

Essa configuração foi observada para todos os anos analisados. Deste modo, a vulnerabilidade à erosão das imagens aéreas, quanto a esse indicador, foi classificada como baixa no setor norte e alta nos demais setores.

\subsubsection{Vegetação}

Através da análise visual das fotografias aéreas do período, observa-se que 1962 é o único ano em que há vegetação densa na regiáo de estudo. A área coberta pela vegetaçáo diminui nos demais anos, e observa-se pouca ou nenhuma vegetação no entorno da praia nos últimos anos analisados.

Desse modo, definiu-se a vulnerabilidade como sendo baixa em todos os setores do ano de 1962. Nos setores do ano de 1973 e nos setores norte e centro do ano de 1977 a vulnerabilidade foi classificada como média. Já para o setor sul do ano de 1977 e para todos os setores dos anos de 1987 e 2001, definiu-se a vulnerabilidade como sendo alta.

\subsubsection{Obras de engenharia costeira}

De acordo com Ceccarelli (2009), desde 2005, obras de engenharia têm sido feitas na praia de Massaguaçú. A presença dessas obras na área de estudo indica uma alta vulnerabilidade à erosão. Entretanto, em relação a esse indicador, o presente estudo não definiu a vulnerabilidade como alta. Isso porque o indicador foi analisado até o ano de 2001, e as obras só foram iniciadas em 2005. Sendo assim, houve ausência de estruturas de contenção costeira no período analisado (1962 - 2001), e a vulnerabilidade à erosão foi classificada como baixa em relação a esse indicador em todos os setores e anos analisados.

\subsection{Integraçáo dos resultados dos indicadores de vulnerabilidade}

A análise de vulnerabilidadese deu através de porcentagens, utilizando como base a Tabela 3. Todos os indicadores tiveram o mesmo peso na análise, e a porcentagem se deu com relação ao número de indicadores analisados para cada ano (oito indicadores para 1962, já que para esse ano não houve análise de posição de linha de costa devido à falta de dados anteriores, e nove indicadores para os demais anos). A evoluçáo das taxas de vulnerabilidades dos setores norte, centro e sul está explicitada na Figura 5.
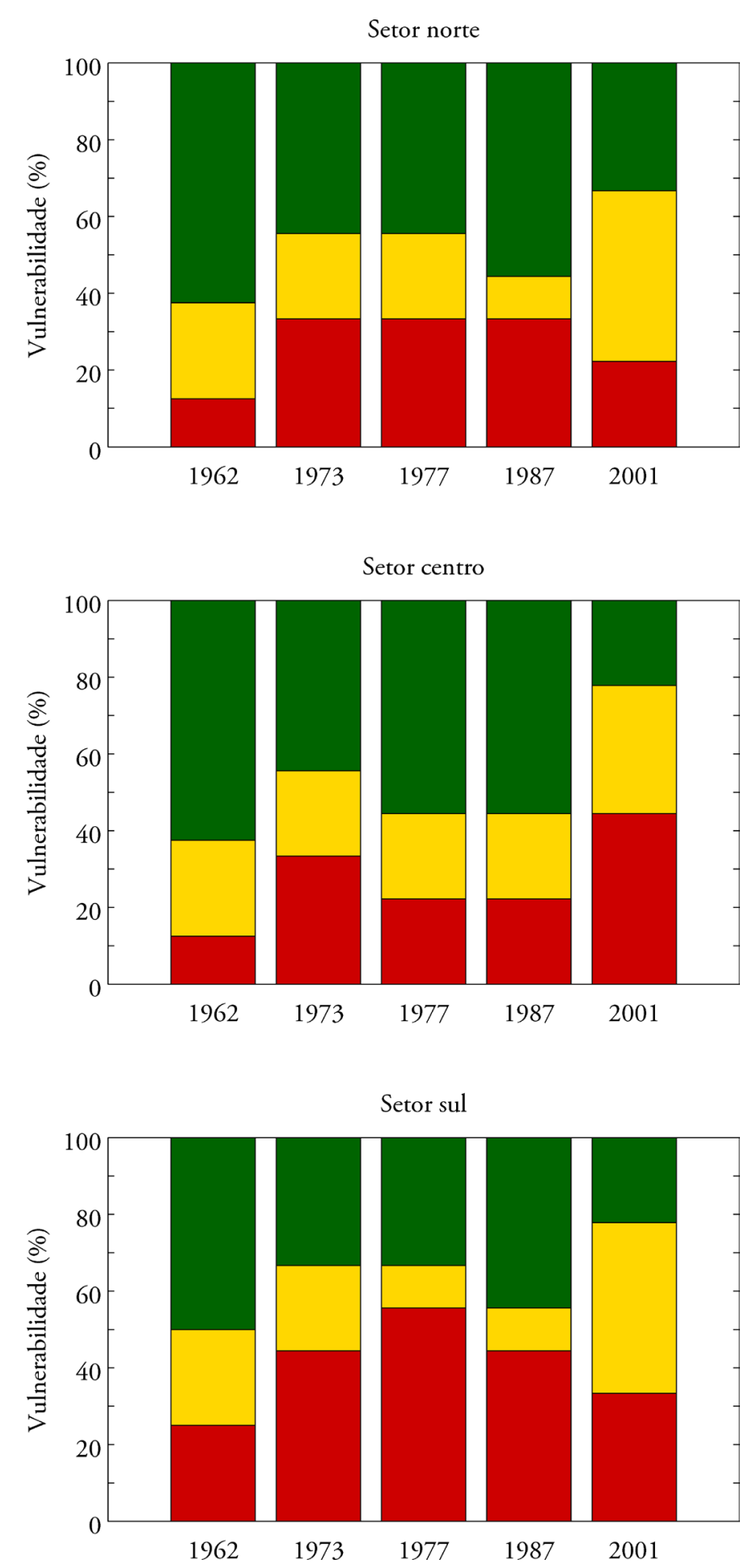

Figura 5. Gráfico ilustrando a evolução da vulnerabilidade na área de estudo. A cor vermelha representa vulnerabilidade alta, a amarela média e a verde, baixa.

Figure 5. Chart of vulnerability temporal evolution in the study area. Red: high vulnerability; yellow: moderate vulnerability; green: low vulnerability. 
No ano de 1962 houve predominância de vulnerabilidade baixa em todos os setores analisados (62,5\% nos setores norte e centro e 50\% no setor sul). No ano de 1973 houve predominância de vulnerabilidade baixa nos setores norte e centro ( $44,4 \%$ nos dois setores) e maior taxa de vulnerabilidade alta no setor sul (44,4\%). Em 1977, os setores norte e centro apresentaram predominância de vulnerabilidade baixa ( $44,4 \%$ e $55,6 \%$, respectivamente) e o setor sul teve maior porcentagem de vulnerabilidade alta (55,6\%). O ano de 1987 apresentou predominância de vulnerabilidade baixa nos setores norte e centro $(55,6 \%)$, e as mesmas porcentagens de vulnerabilidade alta e baixa foram observadas nos setores norte e sul (44.4\%). Já o ano de 2001 apresentou maior porcentagem de vulnerabilidade média nos setores norte e sul $(44,4 \%)$, e predominância de vulnerabilidade alta no setor centro $(44,4 \%) . \mathrm{Na}$ análise do período completo $(1962$ - 2001), percebe-se que as vulnerabilidades alta e média aumentaram em todos os setores, sendo que a alta aumentou principalmente no setor centro (de $12,5 \%$ para $44,4 \%$ ).

\section{DISCUSSÃO}

Analisando a evoluçáo dos resultados para cada setor, percebe-se grande variação nas porcentagens da vulnerabilidade à erosão devido às oscilaçóes da linha de costa e da extensão praial durante o período da avaliação. Nos setores norte e sul a vulnerabilidade aumentou até o ano de 1977, sofreu uma queda em 1987 e voltou a aumentar em 2001. No setor centro a vulnerabilidade também aumentou, mas teve queda nos anos de 1977 e 1987.

Dentre os nove indicadores empregados nesse estudo, quatro deles apresentaram resultados constantes ao longo do tempo (configuraçốes ao largo, presença de rios e/ou desembocaduras, elevação do terreno e obras de engenharia costeira). Quanto aos outros cinco indicadores, três deles (vegetação, taxa de ocupação e permeabilidade do solo) estão intrinsecamente relacionados à urbanização da área de estudo, e apresentaram resultados proporcionais ao aumento da ocupação do local. Somente dois dos indicadores, posição da linha de costa e largura da praia, oscilaram ao longo do tempo, não apresentando um padrão regular de comportamento.

O aumento da ocupação humana teve impacto na regiáo de estudo ao longo dos anos, mas não parece ser um fator determinante ao aumento da vulnerabilidade, uma vez que houve predominância de vulnerabilidades baixas mesmo em anos em que a ocupação esteve crescente. Entretanto, ao se analisar o período entre 1962 e 2001 percebe-se que a vulnerabilidade aumentou principalmente devido ao crescimento da taxa de ocupação e, consequentemente, dos demais indicadores relacionados a ela (vegetação e permeabilidadedosolo). Osmaioresíndices devulnerabilidade ocorreram especialmente no setor centro (a vulnerabilidade alta à erosão cresceu 31,9\%), fato corroborado pelo intenso processo erosivo que tem afetado diretamente a rodovia presente neste setor. Com base nos estudos de Rogacheski (2010) e Luca (2011), acredita-se que a erosão severa em Massaguaçú tem suas causas advindas do vetor de transporte de deriva litorânea, uma vez que a corrente retira sedimentos da parte central da praia depositando-os nos limites norte e sul.

O método para a avaliação do ambiente utilizado no estudo é um procedimento rápido e de baixo custo para a avaliação do ambiente, tornando-o acessível para os gestores dos ambientes costeiros realizarem avaliações rápidas do meio. Conhecer as áreas mais vulneráveis à erosão auxilia os gestores nas tomadas de decisão e no melhor planejamento da orla costeira. Estudos como o proposto neste trabalho são importantes, principalmente no atual cenário de mudanças climáticas globais, uma vez que, com o provável aumento do nível médio do mar, as cidades litorâneas devem se planejar para mitigarem as consequências de alagamentos, intrusão de água salina e outros tantos problemas decorrentes da mudança do padrão climático.

\section{CONCLUSÓES}

A vulnerabilidade à erosão costeira apresentou um comportamento oscilatório devido às variaçóes dos indicadores largura da praia e posição da linha de costa. Apesar disso, houve tendência de crescimento da vulnerabilidade durante o período analisado em razão dos indicadores taxa de ocupação, vegetação e permeabilidade do solo; esse aumento foi mais acentuado no setor central.

Apesar de a ocupação humana ser um influente fator na estrutura e no equilíbrio costeiro, a mesma náo contribui efetivamente para o processo erosivo em Massaguaçú. A maior contribuição para o agravamento da erosão na área de estudo parece ser o comportamento da corrente de deriva litorânea, que transporta os sedimentos da parte central da praia para os setores vizinhos, como sugerido por Rogacheski (2010) e Luca (2011).

Para estudos futuros, sugere-se uma análise quantitativa da vulnerabilidade da regiáo, para que seja possível estimar a magnitude das mudanças na costa ocasionadas pelo provável aumento do nível médio do mar.

\section{AGRADECIMENTOS}

Os autores agradecem à FAPESP (Fundação de Amparo à Pesquisa do Estado de São Paulo) e ao CNPq (Conselho Nacional de Desenvolvimento Científico e Tecnológico) pelo suporte financeiro.

\section{REFERÊNCIAS BIBLIOGRÁFICAS}

Araujo, R.S.; Silva, G.V.; Freitas, D.; Klein, A.H.F. (2009) - Georreferenciamento de fotografias aéreas e análise da variação da linha de costa. In: J. Alcántara-Carrió, I.D. Correa, F. Ila, M. Alvarafo, A.H.F. Klein, J.A. Cabrera \& R. Sandoval (eds.), Métodos en Teledetección Aplicada a la Prevención de Riesgos Naturales en el Litoral, pp.123-138, Cádiz, Espanha. ISBN: 978-84-96023-67-3. Disponível em:http://www.rc.unesp.br/igce/planejamento/download/ isabel/sist_inf_geogr_aula5/foto_aerea_cap8.pdf

Boak, E.H.; Turner, I.L. (2005) - Shoreline Definition and Detection: A Review. Journal of Coastal Research (ISSN: 0749-0208), 21(4):688-703, West Palm Beach, FL, USA. Disponível em http://www6.hawaii.gov/dlnr/occl/ files/Shoreline/JCR-VOL21-4.pdf 
Bosom, E.; Jiménez, J.A. (2011) - Probabilistic coastal vulnerability assessment to storms at regional scale e application to Catalan beaches (NW Mediterranean). Natural Hazards and Earth System Science, 11:475-484. DOI: 10.5194/nhess-11-475-2011

Bush, D.M.; Neal, W.J.; Young, R.S.; Pilkey, O.H. (1999) - Utilization of geoindicators for rapid assessment of coastal-hazard risk and mitigation. Ocean \& Coastal Management, 42(8):647-670. DOI: 10.1016/S09645691(99)00027-7

Cai, F.; Su, X.; Liu, J.; Li, B.; Lei, G. (2009) - Coastal erosion in China under the condition of global climate change and measures for its prevention. Progress in Natural Science, 19(4):415-426. DOI: 10.1016/j.pnsc.2008.05.034.

Ceccarelli, T.S. (2009) - Paradigmas para os projetos de obras maritimas no contexto das mudanças climáticas. $125 \mathrm{p}$., Dissertação de Mestrado, Universidade de São Paulo, São Paulo, SP, Brasil. Não publicado.

Fatorić, S.; Chelleri, L. (2012) - Vulnerability to the effects of climate change and adaptation: The case of the Spanish Ebro Delta. Ocean \& Coastal Management, 60: 1-10. DOI: $10.1016 /$ j.ocecoaman.2011.12.015

INPE(2008) - TOPODATA-Banco de Dados Geomorfométricos do Brasil [Portal internet]. Instituto Nacional de Pesquisas Espaciais (INPE), São José dos Campos, SP, Brasil. In: http://www.dsr.inpe.br/topodata/index.php

IPCC. (2007) - Summary for Policymakers. In: Palutikof, J., Van der Linden, P., Hanson, C. (eds.), Climate Change 2007: Impacts, Adaptation and Vulnerability. Contribution of Working Group II to the Fourth Assessment Report of the Intergovernmental Panel on Climate Change, pp. 7-22, IPCC - Intergovernmental Panel on Climate Change, Cambridge University Press, Cambridge, U.K.

Júnior, J.B.T.; Ouverney, M.I.M.; Ubert, M.S.; Antunes, M.A.H. (2006) - Avaliaçóes de Imagens Ikonos II e QuickBird para obtenção de Bases Cartográficas para cadastro técnico municipal. In: Congresso Brasileiro de Cadastro Técnico Multifinalitário. Anais. Florianópolis, SC, Brasil. Disponível em http://www.ufrrj.br/institutos/ it/deng/marlene/downloads/Artigos/COBRAC\%20 2006\%202.pdf

Linnekamp, F.; Koedam, A.; Baud, I.S.A. (2011) Household vulnerability to climate change: Examining perceptions of households of flood risks in Georgetown and Paramaribo. Habitat International, 35(3):447-456. DOI: $10.1016 /$ j.habitatint.2010.12.003

Luca, C.B. (2011) - Implementaçâo de ferramentas numéricas e bases de dados no SMC-Brasil e sua aplicação no estudo piloto da praia de Massaguaçu-Brasil. 213p., Dissertação de Mestrado, Universidade de Cantabria, Santander, Espanha. Não publicado.
Muehe, D. (2005) - Aspectos gerais da erosão costeira no brasil. Revista Mercator (ISSN: 1984-2201), 4(7):97110, Fortaleza, CE, Brasil. Disponível em http://www. mercator.ufc.br/index.php/mercator/article/view/113/85

Muehe, D. (org). (2006) - Erosão e progradação no litoral brasileiro. 476 p., Instituto Brasileiro do Meio Ambiente e dos Recursos Naturais Renováveis, Brasília, DF, Brasil. ISBN: 85-7738-028-9.

Nicholls, R. (2004) - Coastal flooding and wetland loss in the 21st century: changes under the SRES climate and socio-economic scenarios. Global Environmental Change, 14(1):69-86. DOI: 10.1016/j.gloenvcha.2003.10.007

Nicholls, R.J.; Cazenave, A. (2010) - Sea-level Rise and its impact on coastal zones. Science, 328(5985):1517-1520. DOI: $10.1126 /$ science. 1185782

Pendleton, E.A.; Barras, J.A.; Williams, S.J.; Twichell, D.C. (2010) - Coastal Vulnerability Assessment of the Northern Gulf of Mexico to Sea-level rise and Coastal Change. 26p., U.S. Geological Survey, Open-File Report 20101146, Reston, Virginia, USA. http://pubs.usgs.gov/ of/2010/1146/pdf/ofr2010-1146.pdf

Pianca, C.; Mazzini, P.L.F.; Siegle, E. (2010) - Brazilian offshore wave climate based on NWW3 reanalysis. Brazilian Journal of Oceanography, 58(1):53-70. DOI: 10.1590/S1679-87592010000100006

Rahmstorf, S. (2007) - A semi-empirical approach to projecting future sea-level rise. Science, 315(5810):368370. DOI: $10.1126 /$ science. 1135456

Rogacheski, C.E. (2010) - A Dinâmica Sedimentar e a Caracterização de Zonas de Erosão Acentuada (ZEA) ao Longo do Arco Praialde Massaguaçu, SP. 168p., Dissertação de Mestrado, Universidade de São Paulo, São Paulo, SP, Brasil. Não publicado.

Sousa, P.H.G.O.; Siegle, E.; Tessler, M.G. (2013) Vulnerability assessment of Massaguaçú Beach (SE Brazil). Ocean and Coastal Management, 77:23-40. DOI: 10.1016/j.ocecoaman.2012.03.003

Sousa, P.H.G.O.; Siegle, E.; Tessler, M.G. (2011) Environmental and Anthropogenic Indicators for Coastal Risk Assessment at Massaguaçú Beach (SP) Brazil. Journal of Coastal Research (ISSN: 0749-0208), SI64:319-323, Szczecin, Poland. Disponível em http://www.ics2011.pl/ artic/SP64_319-323_P.H.G.O.Sousa.pdf

Souza, C.R.G.; Luna, G.C. (2010) - Variação da linha de costa e balanço sedimentar de longo período em praias sob risco muito alto de erosáo do município de Caraguatatuba (Litoral Norte de São Paulo, Brasil). Revista de Gestão Costeira Integrada, 10(2):179-199. DOI: $10.5894 /$ rgci176 